OPEN ACCESS

Edited by:

Micaela Sgorbini,

University of Pisa, Italy

Reviewed by:

Irene Nocera,

University of Pisa, Italy

Riccardo Rinnovati,

University of Bologna, Italy

${ }^{*}$ Correspondence:

Lynn Pezzanite

lynn.pezzanite@colostate.edu

Specialty section:

This article was submitted to Comparative and Clinical Medicine,

a section of the journal

Frontiers in Veterinary Science

Received: 06 March 2021

Accepted: 06 April 2021

Published: 21 May 2021

Citation:

Pezzanite L, Chow L, Hendrickson D,

Gustafson DL, Russell Moore A

Stoneback J, Griffenhagen GM,

Piquini G, Phillips J, Lunghofer P,

Dow S and Goodrich LR (2021)

Evaluation of Intra-Articular Amikacin

Administration in an Equine

Non-inflammatory Joint Model to

Identify Effective Bactericidal

Concentrations While Minimizing

Cytotoxicity. Front. Vet. Sci. 8:676774.

doi: 10.3389/fvets.2021.676774

\section{Evaluation of Intra-Articular} Amikacin Administration in an Equine Non-inflammatory Joint Model to Identify Effective Bactericidal Concentrations While Minimizing Cytotoxicity

\author{
Lynn Pezzanite ${ }^{1 *}$, Lyndah Chow ${ }^{1}$, Dean Hendrickson ${ }^{1}$, Daniel L. Gustafson ${ }^{1}$, \\ A Russell Moore ${ }^{2}$, Jason Stoneback ${ }^{3}$, Gregg M. Griffenhagen ${ }^{1}$, Gabriella Piquini ${ }^{1}$, \\ Jennifer Phillips ${ }^{1}$, Paul Lunghofer ${ }^{1}$, Steven Dow ${ }^{1,2}$ and Laurie R. Goodrich ${ }^{1}$
}

\begin{abstract}
${ }^{1}$ Department of Clinical Sciences, College of Veterinary Medicine and Biomedical Sciences, Colorado State University, Fort Collins, CO, United States, ${ }^{2}$ Department of Microbiology, Immunology and Pathology, College of Veterinary Medicine and Biomedical Sciences, Colorado State University, Fort Collins, CO, United States, ${ }^{3}$ Department of Orthopedic Surgery, University of Colorado School of Medicine, Aurora, CO, United States
\end{abstract}

Septic arthritis causes significant morbidity and mortality in veterinary and human clinical practice and is increasingly complicated by multidrug-resistant infections. Intra-articular (IA) antibiotic administration achieves high local drug concentrations but is considered off-label usage, and appropriate doses have not been defined. Using an equine joint model, we investigated the effects of amikacin injected at three different doses (500, 125, and $31.25 \mathrm{mg}$ ) on the immune and cartilage responses in tibiotarsal joints. Synovial fluid (SF) was sampled at multiple time points over $24 \mathrm{~h}$, the cell counts determined, and amikacin concentrations measured by liquid chromatography-mass spectrometry. Cytokine concentrations and collagen degradation products in SF were measured by ELISA and multiplex immunoassays. The mean amikacin concentrations in SF were greater than or equal to the minimum inhibitory concentration (MIC) (0.004 $\mathrm{mg} / \mathrm{ml}$ ) for most common equine joint pathogens at all time points tested to $24 \mathrm{~h}$ for all three amikacin doses evaluated. The inflammatory cytokines tumor necrosis factor-alpha (TNF- $\alpha$ ) and interleukin-1 beta (IL-1 $\beta$ ) increased significantly in SF in the highest amikacin dose group, despite the fact that increases in SF cell counts were not observed. Similarly, the biomarkers of cartilage type II collagen cleavage (C2C and $\mathrm{C} 12 \mathrm{C}$ ) were increased in SF following amikacin injection. Mechanistically, we further demonstrated using in vitro studies that chondrocytes and synoviocytes killed by exposure to amikacin underwent apoptotic cell death and were phagocytosed by macrophages in a non-inflammatory process resembling efferocytosis. Neutrophils and T cells were susceptible to amikacin cytotoxicity at clinically relevant doses, which may result in blunting of cellular inflammatory responses in SF and account for the lack of 
increase in total nucleated cell counts following amikacin injection. In summary, decisions on whether to inject cytotoxic antibiotics such as aminoglycosides intra-articularly and what doses to use should take into account the potential harm that antibiotics may cause and consider lower doses than those previously reported in equine practice.

Keywords: intra-articular, antibiotic, aminoglycoside, amikacin, cartilage, horse

\section{INTRODUCTION}

Septic arthritis causes significant morbidity and mortality in both equine and human clinical practice $(1,2)$. The case fatality rates in humans are $11-15 \%$ in monoarticular disease, but increase to $50 \%$ when multiple joints are involved $(1,3-6)$. Reduced functional outcomes and osteomyelitis are reported in $24 \%$ and $8 \%$ of human survivors, respectively (7). The risk factors for developing septic arthritis in people include joint prosthesis, rheumatoid or osteoarthritis, skin disease, previous intra-articular corticosteroid injection, and comorbidities such as diabetes $(1,8-10)$. Regardless of age group or the risk factors present, Staphylococcus aureus is the most common causative agent in humans, followed by other Gram-positive bacteria $(7,9,11)$. The incidence of septic arthritis in people is reportedly increasing, which has been attributed to a progressively aged population, higher number of invasive orthopedic procedures being undertaken, more frequent orthopedic-related infections, and increased use of immunosuppressive therapies $(1,8)$. Therefore, investigation of therapies to improve outcomes in septic arthritis is indicated.

Current treatments for septic arthritis in both human and equine patients include lavage with debridement of purulent material via arthroscopy or, less commonly, arthrotomy, antibiotics administered systemically and by regional perfusion, or, rarely, serial synovial fluid aspirations in patients too unstable to undergo general anesthesia $(1,2)$. However, unlike the case in human orthopedic practice, antibiotics have also been administered intra-articularly (IA) in equine veterinary medicine as an adjunctive therapy to treat septic arthritis for decades (12-16). This route of administration has more recently gained attention in human medical practice, particularly following prosthetic arthroplasty, in an attempt to address the issue of chronic biofilm infections with an increasing incidence of drug-resistant bacteria through achieving high local antibiotic concentrations (17-21). However, intra-articular antibiotic administration is "off-label" in all species, and appropriate intraarticular doses have not been defined, nor potential cytotoxicity with this route of administration investigated, for any of the major classes of antibiotics. Previous studies have demonstrated the potential for antibiotic cytotoxicity in vitro on joint cells from humans and veterinary species $(17,18,22-24)$. Moreover, despite the widespread use of intra-articular antibiotics in equine practices, studies in horses have revealed variable toxicities following IA antibiotic administration depending on the antibiotic and the dose selected (25-32). However, no previous studies have compared antibiotic cytotoxicity or performed in vivo dose titration studies to assess the impact of antibiotics administered IA in any species on joint inflammation and cartilage degradation.

Therefore, we conducted a series of preliminary in vitro investigations to better define the capacity of different antibiotic classes to exert cytotoxic effects on chondrocytes and synovial cells from horses and dogs $(22,23)$. We demonstrated that amikacin, the antibiotic most commonly used intra-articularly in horses (12-14), was the most cytotoxic of the antibiotics evaluated to chondrocytes and rapidly induced apoptotic cell death (23). We built on that work in this study using a combination of in vitro assays and an in vivo equine model of intra-articular antibiotic administration to evaluate the potential impact of the amikacin doses used clinically in equine practice on overall joint health. We determined the pharmacokinetics of amikacin administered at multiple doses and quantified the biomarkers of inflammation and cartilage damage in the synovial fluid. To further assess macrophage responses to joint cells killed by amikacin, we generated equine monocyte-derived macrophage co-cultures and assessed the macrophage secretion of inflammatory cytokines following engulfment of amikacinkilled cells in a process known as efferocytosis. The overall goal of this work was to identify the amikacin doses that could achieve effective bacterial inhibitory concentrations for common joint pathogens while minimizing the induction of cytotoxicity and inflammation in the joint.

\section{MATERIALS AND METHODS}

\section{Horses}

The use of six healthy 4- to 12-year-old Quarter Horses (four geldings, two mares) was approved by the Colorado State University (CSU) Institutional Animal Care and Use Committee (protocol no. 19-9058A). Horses were determined healthy by physical examination and lameness evaluation by board-certified veterinary surgeons (LG and LP) and radiographic examination (four-view) of both tarsi. All horses were sound in the hindlimbs at the trot and lacked radiographic evidence of osteoarthritis of the tarsal joints prior to study enrollment.

\section{Study Design}

This study was implemented with a randomized incomplete block design, with the random assignments being first leg treated (left or right), with two of the four treatments administered to each horse, and then the order that the treatments were administered. A random number generator (random.org) was used to randomize both the initially treated leg as well as which of the two treatments the horses received. Each horse received either amikacin at one of three doses (amikacin sulfate, $250 \mathrm{mg} / \mathrm{ml}$; Teva Pharmaceuticals, Inc.) or lactated Ringer's solution (LRS) 


\section{In Vivo Experimental Design}

A

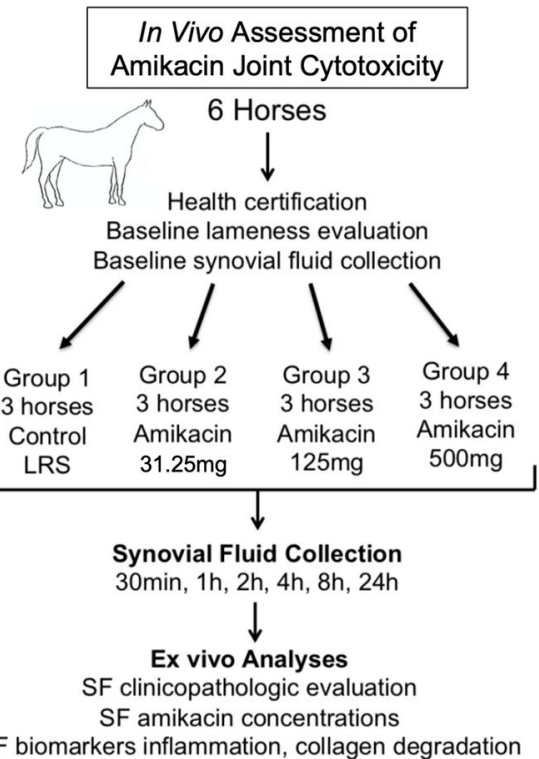

In Vitro Experimental Design

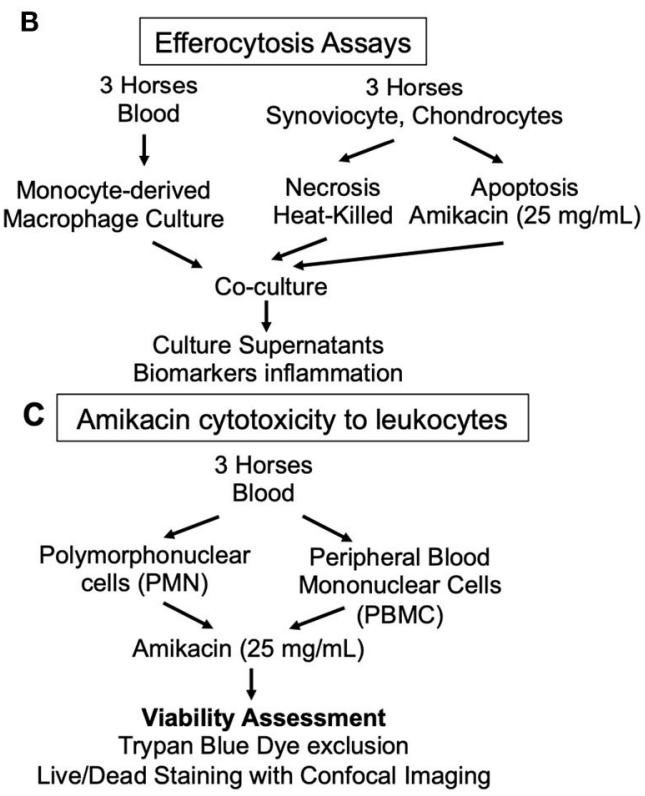

FIGURE 1 | Diagram outlining the experimental design. Schematic overview of the study design for the (A) in vivo assessment of the cytotoxicity of amikacin on the joint of horses; (B) in vitro evaluation of macrophage efferocytosis of joint cells killed by amikacin; and (C) in vitro assessment of amikacin cytotoxicity to leukocytes. SF, synovial fluid.

intra-articularly (Figure 1). The treatment groups were allocated as follows: group 1, $31.25 \mathrm{mg}$ amikacin (diluted in LRS to a total volume of $2 \mathrm{ml}$ ); group 2, $125 \mathrm{mg}$ amikacin (diluted in LRS to a total volume of $2 \mathrm{ml}$ ); group 3, $500 \mathrm{mg}$ amikacin; and group 4, equivalent volume of LRS ( $2 \mathrm{ml})$ as the control group. Each horse was initially assigned to receive one treatment in either the left or the right tibiotarsal joint. After a 2 -week washout period, the contralateral joint was injected with a second randomly assigned treatment. Randomization of treatment allocation and order was performed by one individual (LP) who was unblinded during the experiment; all other collaborators were blinded to the treatments throughout the data collection and analysis. Thus, six horses were administered two treatments each, but no two horses received the same pair of treatments, and each group included three horses in total $(n=3)$. The sample size of three horses per treatment allocation was determined based on previous literature (13).

Horses were sedated with a combination of xylazine $(0.2-$ $1 \mathrm{mg} / \mathrm{kg}$, i.v.) or detomidine (0.01-0.02 $\mathrm{mg} / \mathrm{kg}$, i.v.) and butorphanol tartrate $(0.01 \mathrm{mg} / \mathrm{kg}$, i.v. $)$ to effect. The tibiotarsal joint was aseptically prepared with chlorhexidine gluconate (4\%, VetOne, MWI, Boise, ID, USA) followed by $70 \%$ ethanol and injected as described above. The highest dose was selected based on previous reports by equine practitioners of the use of up to $500 \mathrm{mg}$ amikacin per joint, and lower titrated doses (125 and $31.25 \mathrm{mg})$ were also evaluated $(24,33)$.

\section{Synovial Fluid Sample Collection}

Synovial fluid (SF) samples (1-2 ml) were aspirated from the injected tibiotarsal joint at baseline and at $0.5,1,2,4$,
8 , and $24 \mathrm{~h}$ post-drug administration. Prior to each sample collection, the tibiotarsal joint was aseptically prepared and the horses were sedated as described above. SF was aspirated with a sterile 20-gauge needle into vials and either submitted immediately for clinicopathogical fluid analysis or aliquoted and stored at $-80^{\circ} \mathrm{C}$ until later use in immunoassays or amikacin concentration determination via mass spectrometry for pharmacokinetic analysis.

\section{Synovial Fluid Clinicopathological Parameters}

SF samples were submitted to the CSU Clinical Pathology Laboratory for evaluation by a board-certified clinical pathologist (ARM) for fluid analysis including total nucleated cell count (Hematrue, Heska Corp, Loveland CO, USA), refractometric total protein, preparation of Wright-Giemsa-stained (Aerospray, Logan, UT, USA) direct smears for the determination of a manual leukocyte differential, and subjective grading of the glycosaminoglycans (adequate or disrupted) and quantity of red blood cells (within normal limits or excessive).

\section{Amikacin Concentration Determination}

Extraction and analysis of amikacin from equine SF samples was performed using a high-pressure liquid chromatography/tandem mass spectrometry (LC/MS/MS) system. The system consisted of a 50-mm $\mathrm{C}_{18}$ column (2.1-mm i.d.) with a Phenomenex C18 Filter Frit Guard Cartridge on the Shimadzu HPLC system coupled to the 3200 A-TRAP triple quadrupole mass spectrometer (Applied Biosystems, Inc., Foster City, CA, USA) 
with a flow rate of $750 \mu \mathrm{l} / \mathrm{min}$. The instrument was operated in multiple reaction monitoring (MRM) positive ion mode. The LC gradient conditions (ion-pairing chromatography) were $10 \mathrm{mM}$ heptafluorobutyric acid with $10 \mathrm{mM}$ ammonium hydroxide in Milli-Q water (mobile phase A) and $5 \mathrm{mM}$ heptafluorobutyric acid with $5 \mathrm{mM}$ ammonium hydroxide in 10:90 Milli-Q water/acetonitrile (mobile phase B).

Amikacin stock solution $(100 \mathrm{mg} / \mathrm{ml})$ in $10 \mathrm{mM}$ heptafluorobutyric acid with $10 \mathrm{mM}$ ammonium hydroxide diluted in Milli-Q water was prepared. A standard curve (10, $25,50,75,100,250,500,750,1,000,2,500,5,000$, and 10,000) of amikacin (in micrograms per milliliter) in Milli-Q water was prepared. A 1-mg/ml solution of amikacin in $10 \mathrm{mM}$ ammonium hydroxide in Milli-Q water was prepared, and $40 \mathrm{ml}$ of the final dilution solution (FDS) (50/50) mobile phase B/Milli-Q with $2,500 \mathrm{ng} / \mathrm{ml}$ amikacin was used as an internal standard. Blank equine synovial fluid ( $45 \mu \mathrm{l}$ ) was added to $1.5 \mathrm{ml}$ microcentrifuge tubes as dilution standards, vortexed for $1 \mathrm{~min}$, and centrifuged for $5 \mathrm{~min}$ at $14,000 \mathrm{rpm}$. Standard dilutions (1:100, 1:1,000, and $1: 10,000)$ in Milli-Q water were prepared by transferring $10 \mu \mathrm{l}$ of each standard to $990 \mu \mathrm{l}$ of Milli-Q water in $1.5 \mathrm{ml}$ microcentrifuge tubes. Six QC samples $(3 \times 25,3 \times 100$, and $3 \times 750 \mu \mathrm{g} / \mathrm{ml}$ ) were prepared, vortexed for $5 \mathrm{~min}$, and centrifuged for $1 \mathrm{~min}$ at $8,000 \times g$. Standard samples $(125 \mu \mathrm{l})$ were transferred into sample vials and analyzed.

To analyze the amikacin concentrations in SF samples, dilutions $(1: 100,1: 1,000$, and $1: 10,000)$ were prepared using Milli-Q in $1.5 \mathrm{ml}$ microcentrifuge tubes. The samples were vortex mixed for $5 \mathrm{~min}$, centrifuged for $1 \mathrm{~min}$ at $8,000 \times g$, and transferred into autosample vials for analysis.

\section{Determination of Biomarkers of Cartilage Metabolism}

Competitive ELISAs, previously validated for equine samples (IBEX Pharma, Quebec, Canada), were used to measure the concentrations of biomarkers $\mathrm{C} 2 \mathrm{C}$ (biomarker of type II collagen degradation) and $\mathrm{C} 12 \mathrm{C}$ [biomarker of type I (soft tissue) and type II (cartilage) collagen degradation] in the SF from all treatment groups collected at time points 0,8 , and $24 \mathrm{~h}$ as previously described (34).

\section{Determination of Biomarkers of Joint Inflammation (Cytokines and Collectin)}

The concentrations of C-reactive protein (CRP), a widely accepted inflammatory marker, were measured using competitive ELISA, previously validated for use in equine samples (Immunology Consultant Laboratories, Portland, OR, USA), from all treatment groups at time points 0,8 , and $24 \mathrm{~h}$. A fluorescent bead-based multiplex assay (MILLIPLEX MAP Equine Cytokine/Chemokine Magnetic Beads Multiplex Assay, Millipore Sigma, Burlington, MA, USA) was used to quantify the concentrations of 23 analytes [eotaxin/CCL11, fibroblast growth factor 2 (FGF-2), fractalkine/CS3CL1, granulocyte colony-stimulating factor (G-CSF), granulocyte-macrophage colony-stimulating factor (GM-CSF), GRO, interferon (IFN), interleukin (IL)-1 $\alpha$, IL-1 $\beta$, IL-2, IL-4, IL-5, IL-6, IL-8/CXCL8, IL10, IL-12, IL-13, IL-17a, IL-18, IP-10, MCP-1, RANTES/CCL5, and tumor necrosis factor alpha $(\mathrm{TNF}-\alpha)$ ] in the SF from all time points.

\section{In vitro Culture of Equine Macrophages}

To generate macrophages, equine peripheral blood mononuclear cells were isolated from whole blood of three horses (Quarter Horses, aged 2-3 years, one mare and two geldings) via density gradient centrifugation (Ficoll-Paques ${ }^{\mathrm{TM}}$ PLUS, GE Healthcare Bio-Sciences) and cultured in macrophage medium [Dulbecco's modified Eagle's medium (DMEM) supplemented with $10 \%$ fetal bovine serum, non-essential amino acids, and penicillin/streptomycin antibiotics; Sigma-Aldrich] with human M-CSF (PeproTech, Rocky Hill, NJ, USA) at $25 \mathrm{ng} / \mathrm{ml}$ to stimulate differentiation into macrophages in 3-5 days, as previously described (35).

\section{Efferocytosis and Cytokine Suppression Assays}

Equine synoviocytes and chondrocytes were collected postmortem and isolated from three different horses euthanized for reasons unrelated to the study (Quarter Horses, aged 2-3 years, two geldings and one mare) and expanded in culture as previously described (22). The synoviocytes and chondrocytes were killed rapidly at passage 1 by exposure to either amikacin $(25$ $\mathrm{mg} / \mathrm{ml}$, diluted in DMEM supplemented with $10 \%$ fetal bovine serum and penicillin/streptomycin antibiotics, representing apoptotic cell death) or heat [heat killed (HK), $50^{\circ} \mathrm{C}$ water bath, representing necrotic cell death] and then co-cultured at a 1:1 ratio with equine monocyte-derived macrophages in monolayer culture for $2 \mathrm{~h}$. After $2 \mathrm{~h}$ in culture with dead cells to allow time for phagocytosis, the macrophages were washed in phosphatebuffered saline and allowed to culture in growth media for an additional $18 \mathrm{~h}$. The supernatants were collected at that time for the evaluation of cytokines on a limited panel multiplex bead assay (IL1- $\beta$, IL-6, TNF- $\alpha$; MilliporeSigma, Burlington, MA, USA) and transforming growth factor beta 1 (TGF- $\beta 1$ ) ELISA (R\&D Systems, Minneapolis, MN, USA).

\section{Amikacin Cytotoxicity on Polymorphonuclear Cells and Peripheral Blood Mononuclear Cells}

Three different horses (Quarter Horses, aged 2-3 years, one gelding and two mares) donated blood for the isolation of peripheral blood mononuclear cells (PBMCs) and polymorphonuclear cells (PMNCs), and the cytotoxicity of amikacin on each cell line was assessed. PBMCs were isolated via density gradient centrifugation (Ficoll-Paque ${ }^{\mathrm{TM}}$ PLUS, GE Healthcare Bio-Sciences) at $400 \times g$ for $30 \mathrm{~min}$. PMNCs were isolated using the Lympholyte-Poly (Cedarlane, Peterborough, UK) separation gradient according to the manufacturer's instructions. The PBMCs and PMNs from the three horses were exposed to amikacin over a range of concentrations in complete growth medium $(25,12.5,6.25,3.125,1.56,0.78$, and $0.39 \mathrm{mg} / \mathrm{ml}$ vs. control) in triplicate for 24 or $1 \mathrm{~h}$, respectively. 
Live/dead visualization of PBMCs following amikacin exposure was further performed using the LIVE/DEAD Viability and Counting Kit (Thermo Fisher Scientific) according to the

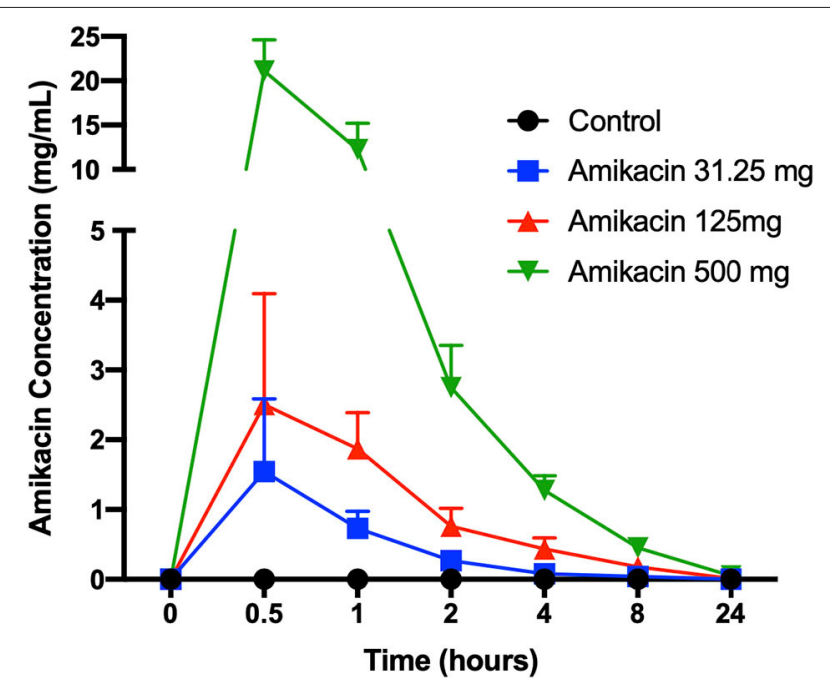

FIGURE 2 | Synovial fluid amikacin concentrations over time following a single intra-articular administration of amikacin at three concentrations. Synovial fluid amikacin concentrations ( $n=3$ horses per group) were determined by high-pressure liquid chromatography/mass spectrometry and expressed as the mean $\pm \mathrm{SD}$. manufacturer's instructions and visualized on an Olympus IC83 confocal microscope. Ratios of live to dead cells were calculated by imaging the total area of each channel using ImageJ software (36).

\section{Data Analysis and Pharmacokinetic Modeling}

Statistical comparisons between the four treatment groups used ANOVA, followed by Tukey's adjustment for multiple comparisons. Data and residuals were visually assessed for normality. All data points collected were included in the final analysis. Significant differences between the clinicopathological parameters and biomarkers were evaluated between baseline and at each time point using a two-way ANOVA with repeated measures. The appearance of glycosaminoglycans, granulated small monocytes, or excessive red blood cells over the range of amikacin concentrations and time was analyzed using logistic regression (function " $\mathrm{glm}$ " from the base STATS package). Biomarkers were normalized to baseline for analysis due to the variability between the baseline values between individual horses.

The half-maximal inhibitory concentration $\left(\mathrm{IC}_{50}\right)$, or the concentration of antibiotic at which $50 \%$ of cells (PBMCs and PMNCs) were viable, was determined by normalizing the dose response for each concentration to the control, transforming the data to normalized dose response vs. $\log 10$ (concentration) and estimating using $\mathrm{IC}_{50}$ non-linear regression by fitting the data to a three-parameter sigmoid function [implemented as " $\log$ (inhibitor) vs. dose response"]. In instances where the $\mathrm{IC}_{50}$

TABLE 1 | Mean \pm SD synovial fluid amikacin concentrations over $24 \mathrm{~h}$ following a single intra-articular administration of amikacin at one of three doses (500, 125, or $31.25 \mathrm{mg}$ ) or the control (LRS, $n=3$ horses per group).

\begin{tabular}{|c|c|c|c|c|c|c|c|c|}
\hline & \multicolumn{8}{|c|}{ Treatment } \\
\hline & Mean $(\mathrm{mg} / \mathrm{ml})$ & SD & Mean $(\mathrm{mg} / \mathrm{ml})$ & SD & Mean $(\mathrm{mg} / \mathrm{ml})$ & SD & Mean $(\mathrm{mg} / \mathrm{ml})$ & SD \\
\hline \multicolumn{9}{|c|}{ Time (h) } \\
\hline 0 & 0.000 & 0.000 & 0 & 0 & 0.000 & 0.000 & 0.000 & 0.000 \\
\hline 0.5 & 0.000 & 0.000 & 1.545 & 1.041 & 2.503 & 1.590 & 21.100 & 3.500 \\
\hline 2 & 0.000 & 0.000 & 0.266 & 0.113 & 0.761 & 0.257 & 2.750 & 0.601 \\
\hline 4 & 0.000 & 0.000 & 0.078 & 0.010 & 0.435 & 0.157 & 1.273 & 0.211 \\
\hline 8 & 0.000 & 0.000 & 0.040 & 0.012 & 0.177 & 0.120 & 0.450 & 0.141 \\
\hline 24 & 0.000 & 0.000 & 0.004 & 0.001 & 0.012 & 0.005 & 0.047 & 0.032 \\
\hline
\end{tabular}

TABLE 2 | Pharmacokinetic parameters evaluated in the synovial fluid following a single intra-articular administration of amikacin at one of three doses to six Quarter Horses.

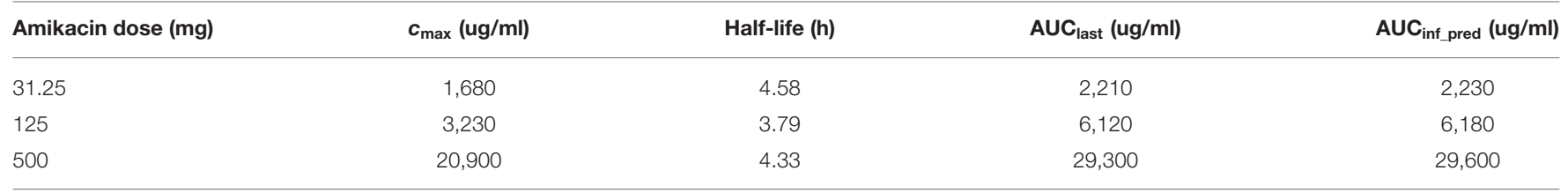

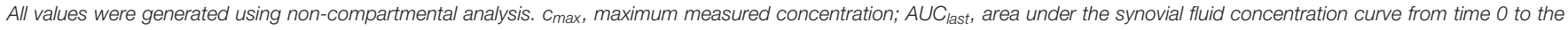
last measurable concentration; $A \cup C_{0-i n f}$, area under the synovial fluid concentration curve extrapolated to infinity. 

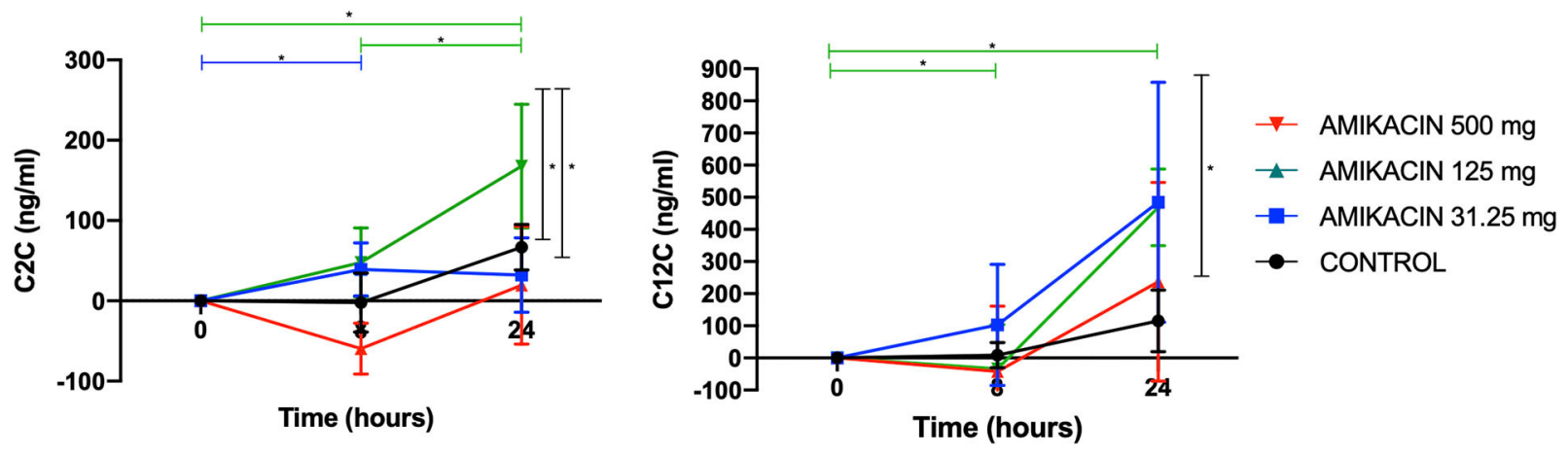

FIGURE 3 | Concentrations of the biomarkers of collagen degradation following a single intra-articular administration of amikacin at three doses compared to the control, normalized to baseline. Competitive enzyme-linked immunosorbent assays (ELISAs), previously validated for use in equine synovial fluid, were used to measure the biomarker concentrations of neo-epitope C2C (biomarker of type II collagen degradation) and C12C [biomarker of type I (soft tissue) and type II (cartilage) collagen degradation]. Results are expressed as the mean $\pm \mathrm{SD}$, normalized to baseline.

was calculated to be outside the range of the concentrations evaluated, or the data were not distributed in a sigmoid fashion following log transformation, the $\mathrm{IC}_{50}$ data were reported as a range of values as the exact value could not be determined based on the concentrations assessed.

The pharmacokinetic parameters for each individual dose level were calculated by non-compartmental analysis using the PKNCA package for $\mathrm{R}$ (37). The ANOVA and $\mathrm{IC}_{50}$ calculations were performed using Prism software v8.4.1 (GraphPad Software Inc., La Jolla, CA, USA). Logistic regression and pharmacokinetic analyses were performed using $\mathrm{R}$ v4.0.0 (“Arbor Day," R Foundation for Statistical Computing, Vienna, Austria). Significance was set at $p \leq 0.05$.

\section{RESULTS}

\section{Defining Amikacin Pharmacokinetics Following Intra-Articular Administration}

Synovial fluid amikacin concentrations were detectable for all doses injected at all time points evaluated, and the mean remained greater than or equal to the minimum inhibitory concentration (MIC) for most common equine pathogens $(>4 \mu \mathrm{g} / \mathrm{ml})$ at $24 \mathrm{~h}$ (Figure 2). The pharmacokinetic data $\left(c_{\max }\right.$, half-life, $\mathrm{AUC}_{\text {last }}$, and $\left.\mathrm{AUC}_{\text {inf_pred }}\right)$ are reported in Table 1. The amikacin values at each time point (mean \pm SD) are reported in Table 2.

\section{Impact of Intra-Articular Amikacin on Biomarkers of Cartilage Metabolism and Health}

Immunoassay revealed dose-dependent increases in the $\mathrm{C} 2 \mathrm{C}$ levels, which were increased in horses treated with $125 \mathrm{mg}$ amikacin at $8 \mathrm{~h}$ vs. baseline $(p=0.02)$, horses receiving $500 \mathrm{mg}$ amikacin at $24 \mathrm{~h}$ vs. baseline $(p=0.01)$, and at 24 vs. $8 \mathrm{~h}(p=$ $0.05)$. When evaluated at each time point, the $\mathrm{C} 2 \mathrm{C}$ levels had a high magnitude of change between the treatment groups at $8 \mathrm{~h}$ for 31.25 vs. $125 \mathrm{mg}(p=0.002)$ and for 125 vs. $500 \mathrm{mg}(p=0.005)$ and at $24 \mathrm{~h}$ for 31.25 vs. $500 \mathrm{mg}(p=0.03)$ and for $125 \mathrm{vs.} 500 \mathrm{mg}$ $(p=0.04)$ (Figure 3).

The $\mathrm{C} 12 \mathrm{C}$ levels increased in horses treated with $500 \mathrm{mg}$ amikacin at $24 \mathrm{~h}$ vs. baseline $(p=0.0006)$ and at 24 vs. $8 \mathrm{~h}$ $(p=0.006)$. When evaluated at each time point, C12C was increased with $500 \mathrm{mg}$ amikacin $v s$. control at $24 \mathrm{~h}(p=0.002)$ (Figure 3).

Glycosaminoglycan content was not different between groups $(p=0.95)$, but was disrupted with increasing frequency over time with repeated arthrocentesis in all treatment groups $(p<0.001)$.

\section{Impact of Intra-Articular Amikacin Administration on Cytokine and Collectin Concentrations in SF}

Cytokine multiplex analysis was used to assess the impact of amikacin on the SF cytokine concentrations over time (Figure 4 and Table 3). CRP concentrations increased in horses treated with $500 \mathrm{mg}$ amikacin compared to all other treatments at 8 and $24 \mathrm{~h}$, which did not reach statistical significance (concentration effect overall, $p=0.08$ ) (Figure 3). Multiplex assay documented detectable levels for 10 of 23 cytokines (IL-1 $\beta$, FGF, G-CSF, IL-10, TNF- $\alpha$, IL-6, IL-8, IL-18, IP-10, or MCP-1). Five cytokines (IL$1 \beta$, FGF, G-CSF, IL-10, and TNF- $\alpha$ ) had different levels between time points for each treatment group evaluated or between treatment groups at different time points (Figure 4 and Table 3 ). IL-1 $\beta$ was higher across time points when $125 \mathrm{mg}$ was injected $v s$. control $(p=0.0008)$ and $500 \mathrm{mg}$ vs. control $(p<0.0001)$. TNF- $\alpha$ levels were higher in samples with $500 \mathrm{mg}$ amikacin $v s$. control $(p=0.03)$. The levels of IL-10 were higher in $31.25 \mathrm{mg}$ amikacin $v s$. control $(p=0.007)$. No differences between treatment groups were observed in the levels of IL-6, IL-8, IL-18, IP-10, or MCP-1.

\section{Synovial Fluid Clinicopathological Parameters}

No differences were found between the treatment groups and controls with respect to total nucleated cell counts, total protein, 

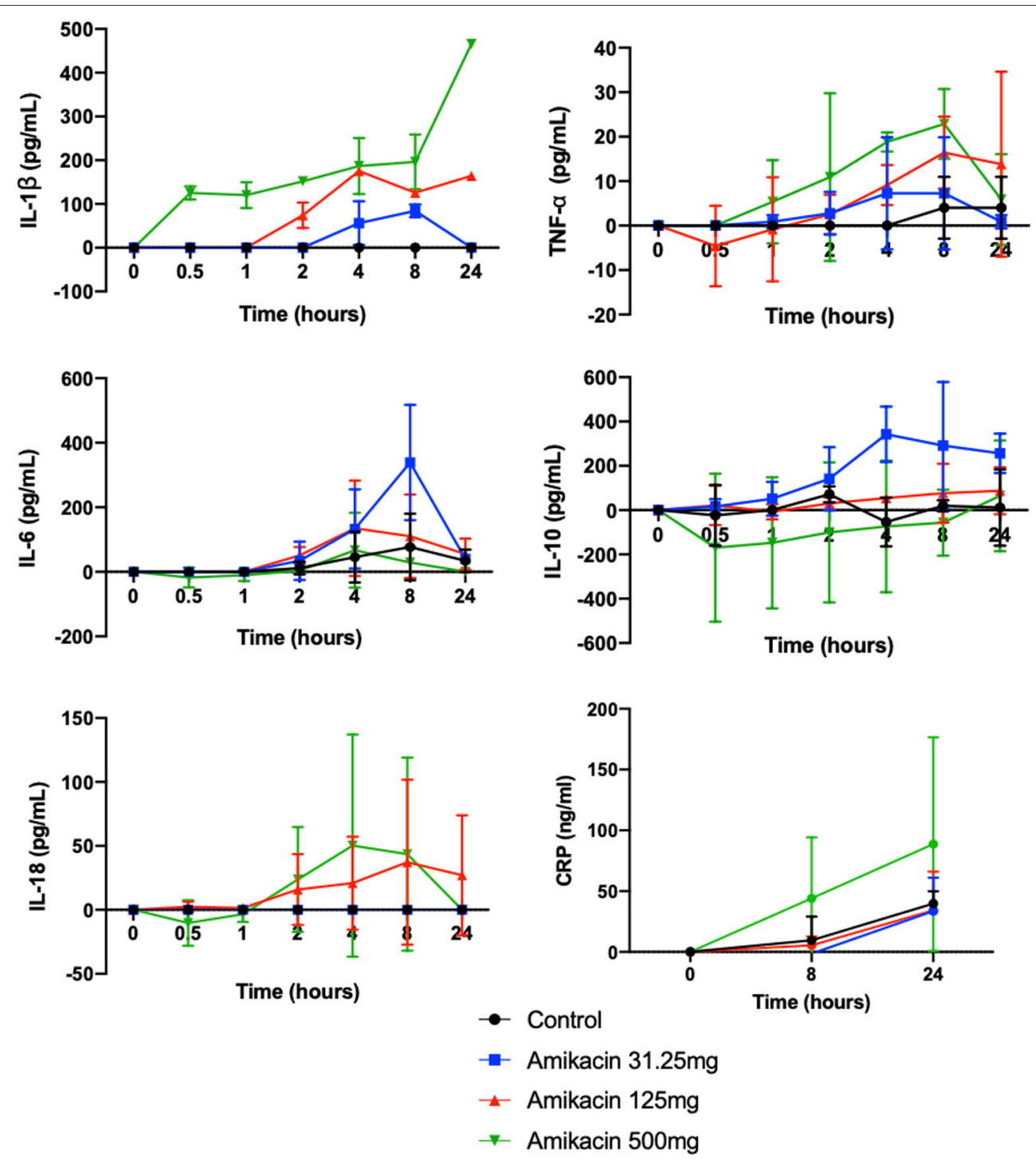

FIGURE 4 | Concentrations of the biomarkers of inflammation in synovial fluid of joints injected with amikacin, normalized to baseline. The concentrations of 23 analytes (eotaxin/CCL11, FGF-2, fractalkine/CS3CL1, G-CSF, GM-CSF, GRO, IFN, IL-1 $\alpha$, IL-1 $\beta$, IL-2, IL-4, IL-5, IL-6, IL-8/CXCL8, IL-10, IL-12 (p70), IL-13, IL-17a, IL-18, IP-10, MCP-1, RANTES/CCL5, and TNF $\alpha$ ) were quantified in synovial fluid samples from all treatment groups at all time points using fluorescent bead-based multiplex assay. Measurable cytokine levels were detectable for 10 of 23 cytokines (IL1 $\beta$, FGF, G-CSF, IL-10, TNF $\alpha$, IL-6, IL-8, IL-18, IP-10, or MCP-1). The cytokine levels were compared between treatment groups at each time point and between time points for each treatment group, as well as for the overall effect of treatment and time by two-way analysis of variance. Significant differences were seen across time points for IL $1-\beta$ for control vs. $125 \mathrm{mg}$ amikacin ( $\rho<0.0008)$ and control vs. $500 \mathrm{mg}(p<0.0001)$, TNF- $\alpha$ for control vs. $500 \mathrm{mg}(p=0.0281)$, and IL-10 for control vs. $31.25 \mathrm{mg}$ amikacin ( $p=0.0066)$. Five cytokines (IL1 $\beta$, FGF, G-CSF, IL-10, and $\mathrm{TNF} \alpha$ ) additionally had significantly different levels between time points for each treatment group evaluated or between treatment groups at different time points. No significant differences between treatment groups were observed in the levels of IL-6, IL-8, IL-18, IP-10, or MCP-1. An ELISA was also used to determine the concentrations of inflammatory C-reactive protein (CRP) in the synovial fluid. Three joints were included per treatment group. The cytokine levels were compared between three treatment groups using a two-way ANOVA. Results are expressed as the mean \pm SD, normalized to baseline.

and red blood cell count at each time point evaluated ( $p$ $=0.35, p=0.70$, and $p=0.14$, respectively) (Figure 5). However, differences were seen between time points with repeated synoviocenteses (total nucleated cell count, $p=0.0002$; total protein, $p=0.001$; red blood cell count, $p=0.02$ ). There were no differences between the amikacin treatments for each of the four cell types (neutrophils, $p=0.14$; monocytes, $p=0.50$; eosinophils, $p=0.89$; basophils, $p=0.14$ ), but there were differences for time with repeated synoviocenteses for each cell line (neutrophils, $p<0.0001$; monocytes, $p<$ 0.0001; eosinophils, $p=0.02$; basophils, $p=0.03$ ) (Figure 5). The presence of granulated small monocytes and excessive red blood cells was not different between treatment groups ( $p=0.41$ and $p=0.05$, respectively). The presence of 
TABLE 3 | Statistical analysis of the cytokine levels in the synovial fluid following amikacin treatment at various doses or the control (lactated Ringer's solution).

\begin{tabular}{|c|c|c|c|c|c|c|c|c|c|c|}
\hline \multirow{2}{*}{$\begin{array}{l}\text { Treatment } \\
\text { Control vs. amikacin } 31.25 \mathrm{mg}\end{array}$} & \multicolumn{2}{|c|}{ IL1- $\beta$} & \multicolumn{2}{|c|}{ TNF- $\alpha$} & \multicolumn{2}{|c|}{ IL-6 } & \multicolumn{2}{|c|}{ IL-10 } & \multicolumn{2}{|c|}{ IL-18 } \\
\hline & ns & 0.10 & ns & 0.78 & ns & 0.35 & * & 0.007 & ns & 0.18 \\
\hline Control vs. amikacin 125 mg & * & 0.0008 & ns & 0.43 & ns & 0.62 & ns & 0.53 & ns & 0.46 \\
\hline Control vs. amikacin 500 mg & * & $<0.0001$ & * & 0.03 & ns & 0.81 & ns & 0.54 & ns & 0.18 \\
\hline
\end{tabular}

Cytokine levels (IL-1 $\beta, T N F-\alpha, I L-6, I L-10$, and IL-18) were compared across time points between treatment groups and the control by two-way ANOVA with repeated measures. Significance was assessed at ${ }^{*} p \leq 0.05$. ns indicates non-significance statistically (significance assessed at $p \leq 0.05$ ). Values indicated are the $p$-values.

granulated small monocytes did not differ over time $(p=$ $0.74)$. The presence of excessive red blood cells increased over time $(p=0.002)$.

\section{Mechanisms of Amikacin Cytotoxicity and Impact on Cell Clearance by Macrophages}

Macrophages engulfing amikacin-killed cells demonstrated a reduced release of pro-inflammatory cytokines (IL-1 $\beta$, TNF$\alpha$, and IL-6) and a concomitant increased production of anti-inflammatory cytokines (TGF- $\beta$ ) (Figure 6). For example, secretion of TGF- $\beta 1$ was increased following efferocytosis of amikacin-killed synovial lining cells compared to necrotic cells $(p=0.01)$ Similarly, amikacin-killed chondrocytes also induced more TGF- $\beta$ than did heat-killed chondrocytes $(p=$ $0.03)$. In contrast, we observed that less IL-1 $\beta$ was produced by macrophages incubated with amikacin-killed cells than by macrophages incubated with heat-killed cells $(p=0.006)$. This was interpreted as evidence of efferocytosis of apoptotic cells, as previously described (38). Similar responses were noted for TNF- $\alpha(p<0.0001)$ and IL-6 $(p<0.0001)$ production between amikacin- vs. heat-killed cells. These findings indicate that cell death induced by amikacin is inherently anti-inflammatory compared to the pathways associated with necrosis. These findings in vitro help explain why SF from amikacin-treated horses manifested a relatively benign response to ongoing cellular death and tissue injury locally within the joint.

\section{Relative Susceptibility of Leukocytes and Joint Cells to Amikacin Cytotoxicity}

The preceding findings suggested another potential mechanism to dampen joint responses to amikacin toxicity. The SF white blood cell counts were not increased in amikacin-treated horses relative to the control, despite evidence of inflammation and cartilage damage (Figures 3-5). This could be explained, for example, if leukocytes, especially neutrophils, were particularly susceptible to amikacin toxicity. Therefore, we determined the mean cytotoxicity-inducing concentrations for purified populations of equine neutrophils and lymphocytes. The $\mathrm{IC}_{50}$ was $6.62 \mathrm{mg} / \mathrm{ml}$ for lymphocytes and between 0.78 and 1.56 $\mathrm{mg} / \mathrm{ml}$ for neutrophils, demonstrating that lymphocyte toxicity would be induced at the concentrations achieved clinically following amikacin injection. Live/dead staining of PBMC following amikacin exposure demonstrated a dose-dependent cytotoxicity of amikacin-exposed PBMC at $4 \mathrm{~h}$ vs. the control (Figure 7). Thus, these findings suggest that the absence of a significant rise in SF leukocytes following amikacin injection may be attributed in part to the direct and rapid leukocyte killing in situ by high concentrations of amikacin, followed by rapid clearance by synovial macrophages.

\section{DISCUSSION}

Intra-articular antibiotics have been used for decades in equine practice and have been more recently reported in humans to treat orthopedic device-related infections following arthroplasty (1921). As all intra-articular antibiotic use is "off-label," appropriate doses have not been determined and potential cytotoxicity by this route of administration has not been fully assessed. Amikacin sulfate is the antibiotic most frequently used in joints in equine clinical practice, and previous work has further demonstrated it to be cytotoxic to equine cartilage in vitro $(22,23)$. For these reasons, we evaluated the cytotoxicity of three doses of amikacin administered in the tarsocrural joint of horses toward the goal of providing dosing guidelines for practitioners, with translational relevance to physicians who may also inject antibiotics in joints. Intra-articular administration of amikacin at doses currently used in horses induced both chondrotoxicity and inflammation in this model. Pharmacokinetic analysis of the synovial fluid indicated that lower amikacin doses than those frequently used in equine practice could achieve effective MICs while minimizing potential toxicity. We further investigated the mechanisms by which amikacin-induced inflammation was resolved within the joint using in vitro co-cultures of equine monocytederived macrophages with chondrocytes and synoviocytes and demonstrated it to be through a process resembling efferocytosis, further suppressing inflammation.

Amikacin sulfate is injected prophylactically in equine joint injections and to treat septic arthritis prior to receipt of bacterial culture and sensitivity due to its broad spectrum of activity against both Gram-positive and Gram-negative bacterial isolates, including Staphylococcus, Escherichia, Enterobacter, and Pseudomonas (39). This study was the first to evaluate an in vivo dose titration for intra-articular amikacin administration in horses, building on previous veterinary literature regarding amikacin pharmacokinetics. Sedrish et al. reported that SF amikacin concentrations remained greater than or equal to the MIC $(4 \mu \mathrm{g} / \mathrm{ml})$ for $24 \mathrm{~h}$ when $500 \mathrm{mg}$ was injected into a normal equine radiocarpal joint (12). Taintor et al. further demonstrated that joint inflammation accelerated amikacin distribution from the joint, as amikacin (500 mg, radiocarpal joint) remained greater than or equal to the MIC in normal joints for $72 \mathrm{~h}$, but only $48 \mathrm{~h}$ in endotoxin-inflamed joints, which was attributed 

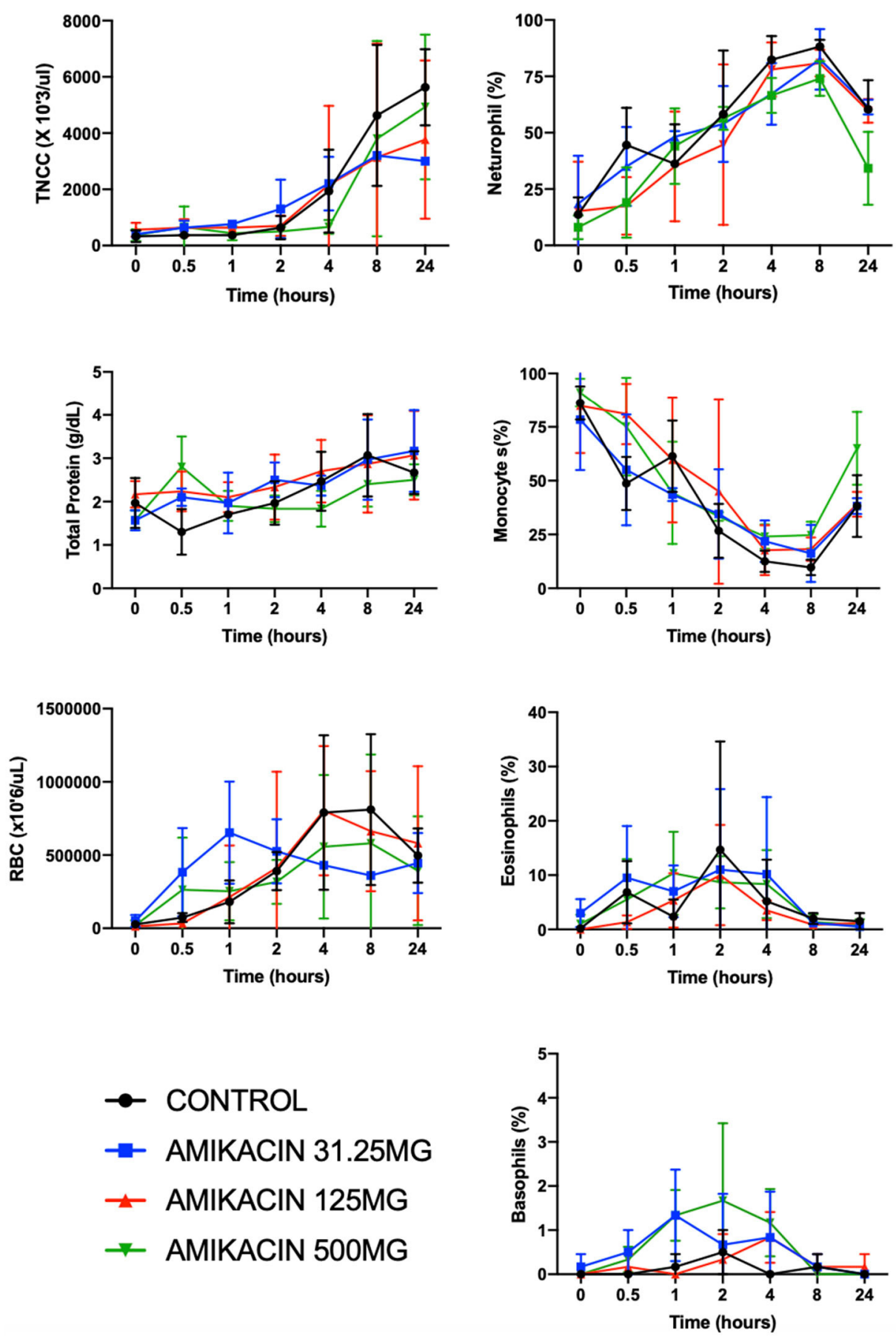

FIGURE 5 | Clinicopathologic parameters for synovial fluid following a single intra-articular administration of amikacin in three treatment groups. Fluid analysis on synovial fluid samples obtained from three horses per group was performed on all samples for total nucleated cell count, total protein, and red blood cell count and leukocyte differential ( $y$-axes are not equivalent across graphs). There were significantly increased neutrophils (in percent) and decreased monocytes (in percent) with repeated arthrocenteses over time. Results are expressed as the mean $\pm \mathrm{SD}$.

to the differential antibiotic movement from the joint with increased synovial vascularity in inflammation (14). In this study, the mean amikacin concentrations in the synovial fluid reached levels well over $100 \times$ the MIC, and the mean levels were sustained at or above the established MIC for most common equine pathogens $(4 \mu \mathrm{g} / \mathrm{ml})$ for at least $24 \mathrm{~h}$ following injection of all the amikacin doses assessed $(31.25,125$, and $500 \mathrm{mg}$ ) (40). While it is recognized that select equine organisms have 


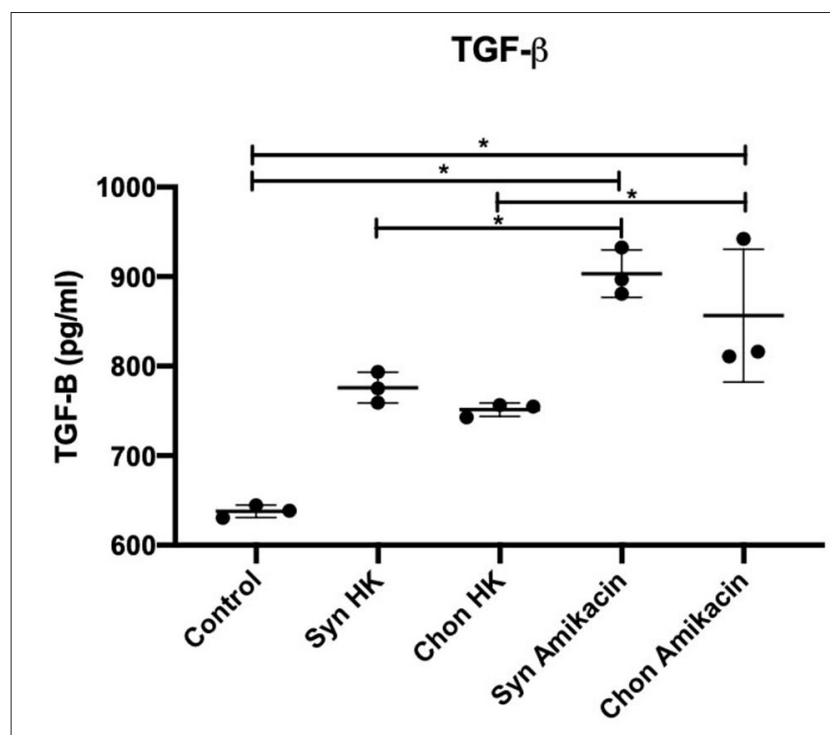

IL-1 $\beta$

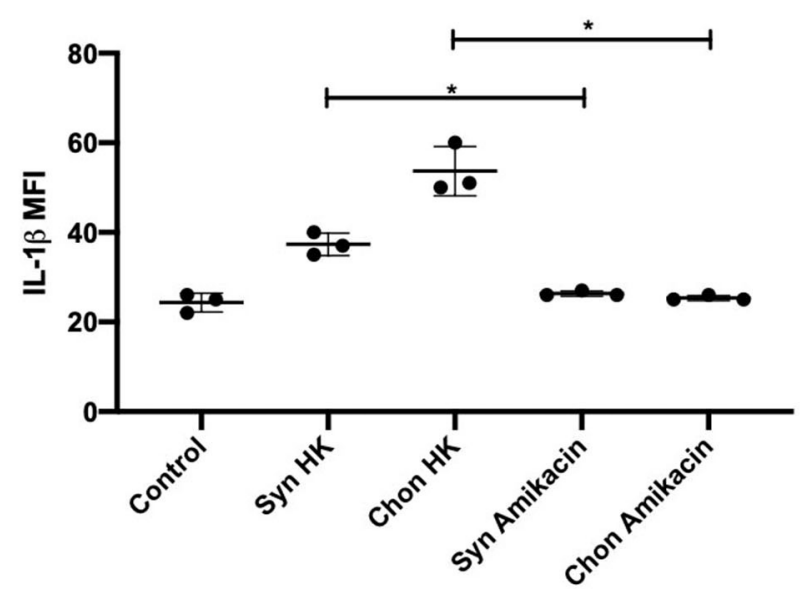

\section{IL-6}

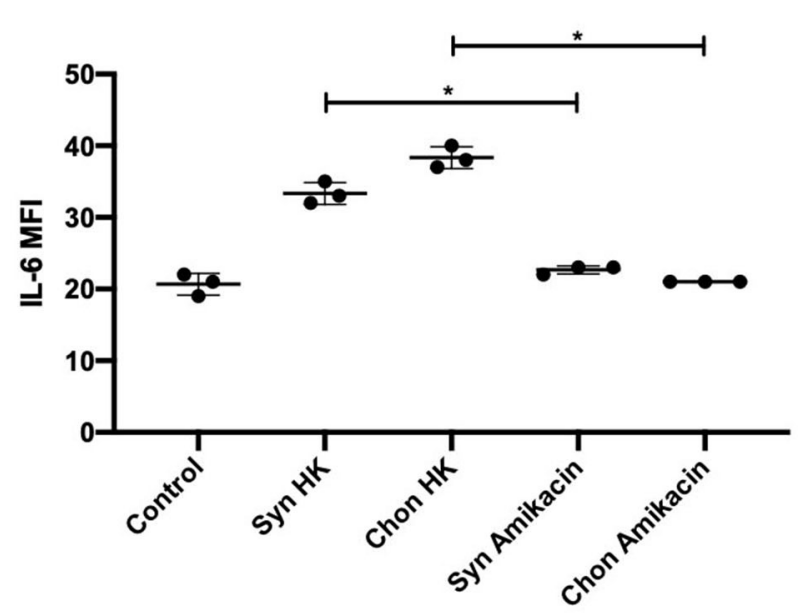

TNF- $\alpha$

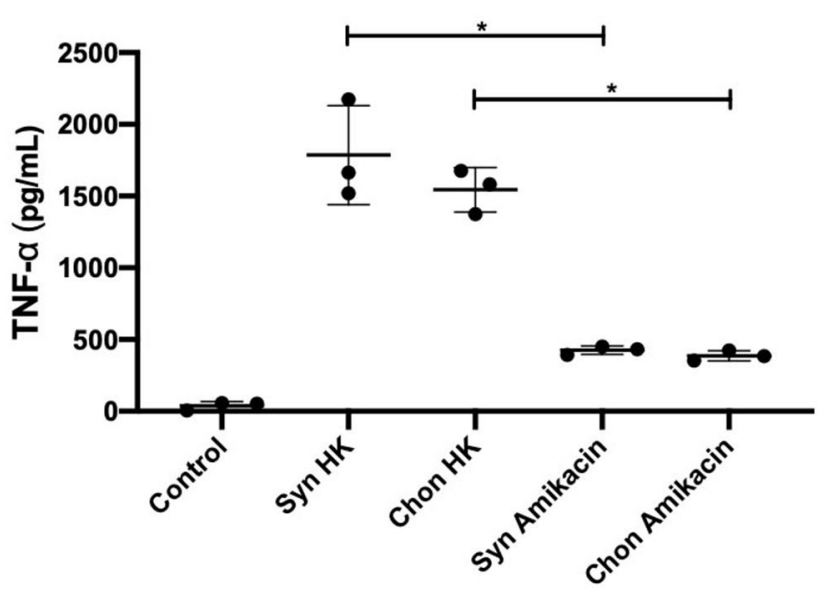

FIGURE 6 | Efferocytosis and cytokine suppression assays quantifying IL1- $\beta$, IL-6, TNF- $\alpha$, and TGF- $\beta 1$ in macrophage culture media after incubation with equine synoviocytes and chondrocytes killed by induced apoptosis ( $25 \mathrm{mg} / \mathrm{ml}$ amikacin) or necrosis (heat killed, HK). Data were evaluated by two-way ANOVA. Significance was assessed at ${ }^{*} p \leq 0.05$.

been reported to have higher MICs (e.g., coagulase-positive Staphylococcus sp., $8 \mu \mathrm{g} / \mathrm{ml}$; Pseudomonas aeruginosa, $64 \mu \mathrm{g} / \mathrm{ml}$; and Streptococcus zooepidemicus, $128 \mu \mathrm{g} / \mathrm{ml}$ ), these findings would suggest that amikacin doses lower than those commonly used by equine practitioners $(125-500 \mathrm{mg}$ ) may be utilized to both reach and sustain therapeutic levels, although further evaluation in inflamed or infected joints is warranted $(14,33)$.

The degree of inflammation induced by amikacin injection and the subsequent mechanisms of resolution of inflammation warrant further discussion. Clinicopathological analysis of synovial fluid samples revealed alterations in differential cellular compositions over time with repeated synoviocenteses, but no differences between treatment groups. This may be attributed to the frequency of SF sampling, resulting in elevated total nucleated cell counts and total protein as well as an altered leukocyte differential, which could have overwhelmed any individual treatment effect. However, amikacin injection elicited dosedependent increases in the pro-inflammatory cytokines TNF- $\alpha$ and IL1- $\beta$ in the joint, indicating an inflammatory response. The concentrations of IL-10 were also elevated in the synovial fluid of horses injected with the lowest dose of amikacin; as IL-10 secretion is typically suppressed by IL- $1 \beta$, this may be attributed to the less significant IL-1 $\beta$ downregulation of IL-10 at lower amikacin concentrations.

We explored this further to determine the mechanism by which amikacin-induced inflammation was resolved using in vitro assays to demonstrate phagocytosis and the clearance of joint cells killed by amikacin via equine macrophages. Dead and 


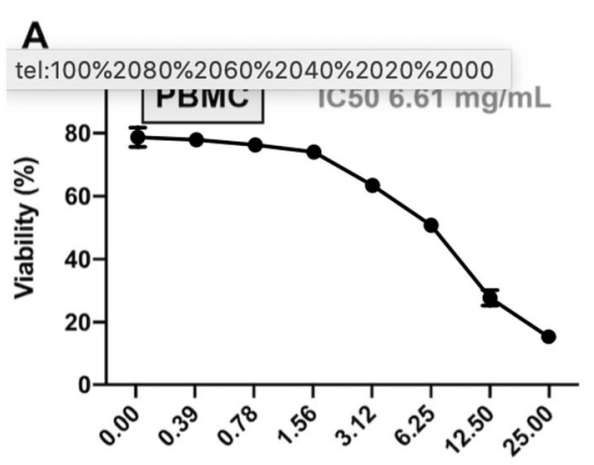

C Amikacin Concentration $(\mathrm{mg} / \mathrm{mL})$
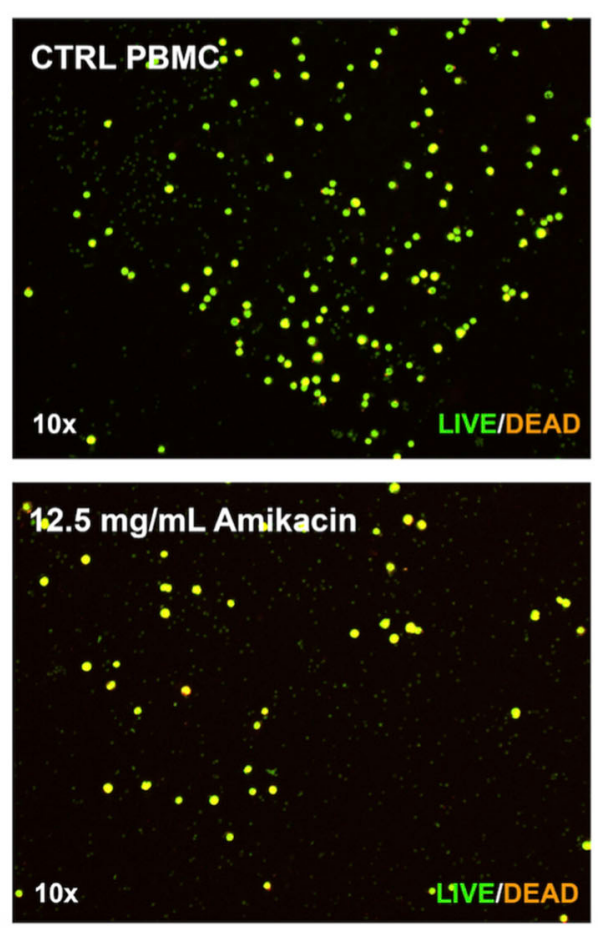

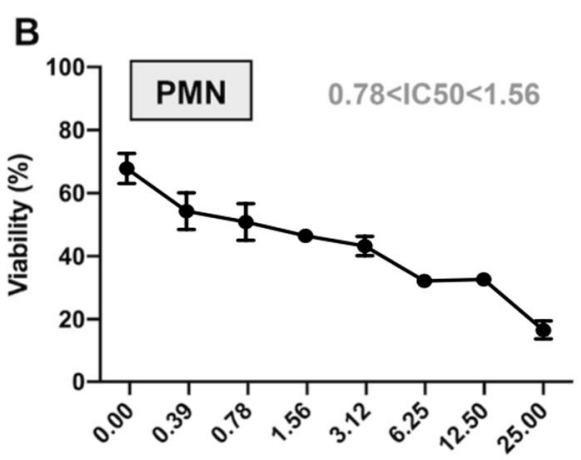

Amikacin Concentration $(\mathrm{mg} / \mathrm{mL})$
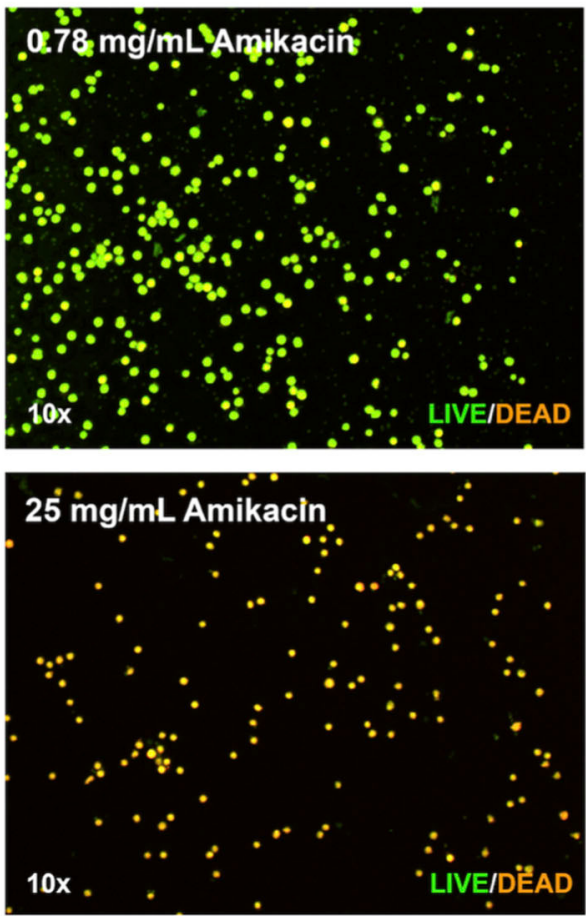

FIGURE 7 | Effect of amikacin on neutrophils and peripheral blood mononuclear cells at clinically relevant doses. Cytotoxicity of amikacin on (A) peripheral blood mononuclear cells (PBMCs) and (B) polymorphonuclear cells (PMNs) derived from whole blood of three horse donors each in triplicate and assessed by Trypan blue dye exclusion over a range of amikacin concentrations. The inhibitory concentration $50\left(\mathrm{IC}_{50}\right)$, or the concentration at which $50 \%$ of the cells were dead, is denoted. (C) Live/dead visualization of PBMCs following amikacin exposure over a range of doses reached clinically $(0.78,12.5$, and $25 \mathrm{mg} / \mathrm{ml})$ performed using the LIVE/DEAD Viability and Counting Kit (Thermo Fisher Scientific).

dying cells are rapidly cleared in the body by tissue macrophages through a process known as efferocytosis, and the mechanism of cell death (e.g., apoptosis and necrosis) significantly impacts how macrophages respond to these phagocytosed cells (38). In general, macrophage phagocytosis of apoptotic cells leads to the suppression of inflammation, while engulfment of necrotic cells provides a strong inflammatory stimulus (41). Therefore, we conducted in vitro experiments to elucidate the mechanisms by which amikacin kills joint cells and then examined the effects on macrophage activation responses following engulfment of dead and dying cells. Stimulation of anti-inflammatory cytokine production (TGF- $\beta$ ) and suppression of pro-inflammatory cytokine secretion (TNF- $\alpha$, IL1- $\beta$, and IL-6) were observed in this model compared to cells that died by necrotic pathways, consistent with previous reports $(38,41)$. Efferocytosis has been described as an important step to resolving inflammation and restoring normal tissue function, with macrophages playing an integral role in the maintenance of joint homeostasis (38, 41). The differential cytotoxicity of amikacin to cell types was also demonstrated, with leukocytes overall, and particularly neutrophils, being sensitive to amikacin-induced cell death at concentrations that would be easily achieved in synovial fluid with the doses commonly used in equine practice. The susceptibility to antibiotic killing of leukocytes likely accounts 
for the lack of differences observed in the clinicopathological parameters of synovial fluid and could mask the inflammatory effects of antibiotic-induced cytotoxicity. These findings indicate that particular attention should be paid to the off-target cytotoxic effects of certain antibiotics when used for local treatment of infections due to the potential for inducing tissue injury and immune suppression.

Limitations to the study design include the small horse sample size, evaluation in normal vs. inflamed joints, lack of synovial fluid collection at later time points for biomarker analysis to investigate when inflammatory cytokine concentrations returned to baseline, and the lack of histopathologic evaluation of synovial tissues. Obtaining a bigger sample size and performing additional sampling of the synovial fluid past $24 \mathrm{~h}$ were not possible due to financial constraints, but may have revealed continued increases in the biomarkers of collagen degradation and potentially statistically significant elevations in the CRP values, as has been previously reported (34). Furthermore, synovial fluid sampling at later time points and evaluation of the histopathology of synovial tissues may have provided further information as to whether intra-articular amikacin administration resulted in long-term joint damage or was only associated with a transient increase in pro-inflammatory cytokines and cartilage degradation products. The timing and frequency of synovial fluid sampling were performed to emphasize pharmacokinetic analysis of amikacin concentrations in the synovial fluid, where a greater number of early time points allowed determining synovial fluid concentration inflection points and the single 24-h time point allowed calculating $\beta$ and the terminal half-life slope, as has been previously described $(13,34)$. Synovial fluid sampling may have transiently affected the other parameters evaluated (e.g., clinicopathological parameters), as has been previously reported (42), but the study design, which included a control sample, should have mitigated this potential complication. Comparison of the elevations in inflammatory biomarkers and cartilage degradation products to long-term histopathological findings may have provided some clarity as to whether the detrimental effects associated with amikacin injection reported here are temporary or enduring, as cytological evaluation of the synovial fluid has been previously reported to be more sensitive than histology (43). Further in vivo evaluation of antibiotic distribution and cytotoxicity when co-administered with other medications intra-articularly or in inflamed or infected joints is warranted. In investigating the use of amikacin in the treatment of infected joints, evaluation of additional parameters not reported here, such as synovial fluid $\mathrm{pH}$ and glucose and lactate levels, may be important in monitoring response to therapy $(44,45)$. This work will serve as a platform from which further investigations of intra-articular antibiotic use will build.

The horse represents a valuable large animal preclinical model for human joint disease, including treatment of septic arthritis (46-53). The joint volume and cartilage thickness of horses more closely approximate that of human cartilage compared to other animal models, and the larger volume of synovial fluid within equine joints allows for a greater ease of synovial fluid sampling for the analysis of increased outcome parameters (46-50). Equine articular cartilage is subject to loading forces of similar or greater magnitude than human cartilage, which may have important implications when evaluating intra-articular therapies (46-48). The formation of bacterial biofilm aggregates in equine synovial fluid as a substitute for human synovial fluid was recently described in vitro, and clinical evidence of septic arthritis as a disease process in horses is well-documented, providing further evidence to support the use of the equine preclinical model as both a translational and clinically relevant model for human joint disease $(52,54)$.

This study demonstrated that the concentrations of amikacin administered intra-articularly in horses reached and sustained the therapeutic levels for most common equine pathogens for all doses assessed while eliciting an increased production of cartilage degradation products and pro-inflammatory biomarkers. The effects of amikacin on equine cartilage in situ and in the presence of inflammation and sepsis warrant further investigation, but these findings indicate that amikacin can penetrate the cartilage to elicit a dose-dependent cytotoxicity in vivo. In conclusion, the use of intra-articular antibiotics has the potential to augment the current treatment regimens for septic arthritis, but induces joint inflammation and cartilage degradation at higher doses in vivo. Decisions on whether to inject cytotoxic antibiotics such as aminoglycosides intra-articularly and what doses to use should take into account the potential harm that antibiotics may cause and consider lower doses than those previously reported in equine practice.

\section{DATA AVAILABILITY STATEMENT}

The raw data supporting the conclusions of this article will be made available by the authors, without undue reservation.

\section{ETHICS STATEMENT}

The animal study was reviewed and approved by Institutional Animal Care and Use Committee of Colorado State University (protocol \#19-9058A).

\section{AUTHOR CONTRIBUTIONS}

LP, LG, SD, LC, DH, and JS conceptualized and designed the study. LP, LG, SD, LC, JP, GP, PL, GG, DG, and AR acquired the data. LP, LG, SD, LC, GG, PL, JP, DG, AR, and JS analyzed and interpreted the data. LP, LC, and GG drafted the manuscript. All authors contributed to and approved the submitted version of the manuscript.

\section{FUNDING}

This study was funded by the Animal Health and Disease (Grant No. NI18AHDR1019785G010) from the USDA National Institute of Food and Agriculture, CCTSI NIH/NCATS CTSA 5TL1TR002533-02, NIH 5T32OD010437-19, Verdad Foundation, Shipley Family Foundation, Carolyn Quan and Bennett Porter, Colorado State University Veterinary Summer Scholars Program, and Colorado State University Young Investigator Award in Companion Animal Studies. 


\section{ACKNOWLEDGMENTS}

The authors would like to thank and acknowledge Jennifer Daniels, Ryan Shelton, Natalie Lombard, and staff of the Orthopaedic Research Center at Colorado State

\section{REFERENCES}

1. Matthews C, Weston V, Jones A, Field M, Coakley G. Bacterial septic arthritis in adults. Lancet. (2010) 375:846-55. doi: 10.1016/S0140-6736(09)61595-6

2. Morton A. Diagnosis and treatment of septic arthritis. Vet Clin North Am Equine Pract. (2005) 21:627-49. doi: 10.1016/j.cveq.2005.08.001

3. Gupta M, Sturrock R, Field M. A prospective 2-year study of 75 patients with adult-onset septic arthritis. Rheumatology. (2001) 40:2430. doi: 10.1093/rheumatology/40.1.24

4. Tong S, Davis J, Eichenberger E, Holland TL, Fowler VG. Staphylococcus aureus infections: epidemiology pathophysiology, clinical manifestations, and management. Clin Microbiol Rev. (2015) 28:603-61. doi: 10.1128/CMR.00134-14

5. Tande A, Patel R. Prosthetic joint infection. Clin Microbiol Rev. (2014) 27:30245. doi: 10.1128/CMR.00111-13

6. Nair R, Schweizer M, Singh N. Septic arthritis and prosthetic joint infections in older adults. Infect Dis Clin North Am. (2017) 31:71529. doi: 10.1016/j.idc.2017.07.013

7. Weston V, Jones A, Bradbury N, Fawthrop F, Doherty M. Clinical features and outcome of septic arthritis in a single UK health district 1982-1991. Ann Rheum Dis. (1999) 58:214-9. doi: 10.1136/ard.58.4.214

8. Gierrson A, Statkevicius S, Vikingsson A. Septic arthritis in Iceland 1990-2002: increasing incidence due to iatrogenic infections. Ann Rheum Dis. (2008) 67:638-43. doi: 10.1136/ard.2007.077131

9. Kaandorp C, Dinant H, van de Laar M, Moens HJ, Prins AP, Dijkmans BA. Incidence and sources of native and prosthetic joint infection: a community based prospective survey. Ann Rheum Dis. (1997) 56:4705. doi: 10.1136/ard.56.8.470

10. Edwards C, Cooper C, Fisher D, Field M, van Staa TP, Arden NK. The importance of the disease process and disease-modifying antirheumatic drug treatment in the development of septic arthritis in patients with rheumatoid arthritis. Arthritis Rheum. (2007) 57:1151-7. doi: 10.1002/art.23003

11. Dubost J, Soubrier M, De Champs C, Ristori JM, Bussiere JL, Sauvezie B. No changes in the distribution of organisms responsible for septic arthritis over a 20-year period. Ann Rheum Dis. (2002) 61:267-9. doi: 10.1136/ard.61.3.267

12. Sedrish S, Moore R, Barker S. Pharmacokinetics of single dose intra-articular administration of amikacin in the radiocarpal joints of normal horses. In: Proceedings of the American College of Veterinary Surgeons Meeting, Chicago, Illinois (1994). p. 437.

13. Orsini J, Soma L, Rourke J, Park M. Pharmacokinetics of amikacin in the horse following intravenous and intramuscular administration. J Vet Pharm Ther. (1985) 8:194-201. doi: 10.1111/j.1365-2885.1985.tb00944.x

14. Taintor J, Schumacher J, DeGraves F. Comparison of amikacin concentrations in normal and inflamed joints of horses following intra-articular administration. Equine Vet J. (2006) 38:18991. doi: 10.2746/042516406776563233

15. Lloyd K, Stover S, Pascoe J, Baggot JD, Kurpershoek C, Hietala S. Plasma and synovial fluid concentrations of gentamicin in horses after intra-articular administration of buffered and unbuffered gentamicin. Am J Vet Res. (1988) 49:644-9.

16. Werner L, Hardy J, Bertone A. Bone gentamicin concentration after intraarticular or regional intravenous perfusion in the horse. Vet Surg. (2003) 32:559-65. doi: 10.1111/j.1532-950X.2003.00559.x

17. Dogan M, Isyar M, Yilmaz I. Are the leading drugs against Staphylococcus aureus really toxic to cartilage? J Infect Public Health. (2016) 9:2518. doi: 10.1016/j.jiph.2015.10.004

18. Edin M, Miclau T, Lester G, Lindsey RW, Dahners LE. Effect of cefazolin and vancomycin on osteoblasts in vitro. Clin Orthop Relat Res. (1996) 333:24551. doi: 10.1097/00003086-199612000-00027
University for the care of university-owned research horses and assistance with the procedures described. The authors would further like to acknowledge Dr. Ann Hess, Professor of Statistics, for her assistance with data analysis.
19. Whiteside L, Roy M, Nayfeh T. A direct approach to treatment of infected total knee arthroplasty. Bone Joint J. (2016) 98:326. doi: 10.1302/0301-620X.98B.36276

20. Peppers M, Whiteside L, Lazear R, Roy M. Vancomycin injected in the knee achieved high synovial fluid concentration with a half-life of 3.4 hours. In: Proceedings, Orthopedic Research Society. Long Beach, CA (2011).

21. Whiteside L, Roy M. One-stage revision with catheter infusion of intraarticular antibiotics successfully treats infected THA. Clin Orthop Relat Res. (2017) 475:419-29. doi: 10.1007/s11999-016-4977-y

22. Pezzanite L, Chow L, Soontararak S, Phillips J, Goodrich L, Dow S. Amikacin induces rapid dose-dependent apoptotic cell death in equine chondrocytes and synovial cells in vitro. Equine Vet J. (2020) 52:71524. doi: $10.1111 /$ evj.13243

23. Pezzanite L, Chow L, Piquini G, Griffenhagen G, Ramirez D, Dow S, et al. Use of in vitro assays to identify antibiotics that are cytotoxic to normal equine chondrocytes and synovial cells. Equine Vet J. (2020) 53:57989. doi: 10.1111/evj.13314

24. Antoci V, Adams C, Hickok N, Shapiro IM, Parvizi J. Antibiotics for local delivery systems cause skeletal cell toxicity in vitro. Clin Orthop Relat Res. (2007) 462:200-6. doi: 10.1097/BLO.0b013e31811ff866

25. Mills ML, Rush BR, Jean GS, Gaughan EM, Mosier D, Gibson E, et al. Determination of synovial fluid and serum concentrations, and morphologic effects of intraarticular ceftiofur sodium in horses. Vet Surg. (2000) 5:398406. doi: 10.1053/jvet.2000.9141

26. Newman J, Prange T, Jennings S, Barlow BM, Davis JL. Pharmacokinetics of tobramycin following intravenous, intramuscular, and intra-articular administration in healthy horses. J Vet Pharmacol Ther. (2013) 36:53241. doi: $10.1111 /$ jvp. 12048

27. Sanchez Teran A, Rubio-Martinez L, Villarino N, Sanz M. Effects of repeated intra-articular administration of amikacin on serum amyloid A, total protein and nucleated cell count in synovial fluid from healthy horses. Equine Vet J Suppl. (2012) 43:12-6. doi: 10.1111/j.2042-3306.2012.00637.x

28. Stover S, Pool R. Effect of intra-articular gentamicin sulfate on normal equine synovial membrane. Am J Vet Res. (1985) 46:2485-91.

29. Lescun T, Adams S, Wu C, Bill RP, Van Sickle DC. Effects of continuous intra-articular infusion of gentamicin on synovial membrane and articular cartilage in the tarsocrural joint of horses. Am J Vet Res. (2002) 63:6837. doi: 10.2460/ajvr.2002.63.683

30. Davenport C, Boston R, Richardson D. Effects of enrofloxacin and magnesium deficiency on matrix metabolism in equine articular cartilage. Am J Vet Res. (2001) 62:160-6. doi: 10.2460/ajvr.2001.62.160

31. Egerbacher $M$, Edinger J, Tschulenk W. Effects of enrofloxacin and ciprofloxacin hydrochloride on canine and equine chondrocytes in culture. Am J Vet Res. (2001) 62:704-8. doi: 10.2460/ajvr.2001.62.704

32. Bolt D, Ishihara A, Weisbrode S, Bertone A. Effects of triamcinolone acetonide, sodium hyaluronate, amikacin sulfate, and mepivacaine hydrochloride, alone and in combination, on morphology and matrix composition of lipopolysaccharide-challenged and unchallenged equine articular cartilage explants. Am J Vet Res. (2008) 69:861-7. doi: 10.2460/ajvr.69.7.861

33. Bohannon L, Owens S, Walker N, Carrade DD, Galuppo LD, Borjesson DL. The effects of therapeutic concentrations of gentamicin, amikacin, and hyaluronic acid on cultured bone marrow derived equine mesenchymal stem cells. Equine Vet J. (2013) 45:732-6. doi: 10.1111/evj. 12045

34. Knych H, Mama K, Moore C, Hill AE, McKemie DS. Plasma and synovial fluid concentrations and cartilage toxicity of bupivacaine following intraarticular administration of liposomal formulation to horses. Equine Vet J. (2019) 51:408-14. doi: 10.1111/evj.13015 
35. Ma J, Wang S, Lin Y, Liu H, Liu Q, Wei H, et al. Infection of equine monocyte-derived macrophages with an attenuated equine infectious anemia virus (EIAV) strain induces a strong resistance to the infection by a virulent EIAV strain. Vet Res. (2014) 45:82. doi: 10.1186/s13567-0140082-y

36. Rasband W. Image J, U.S. (1997-2018). Bethesda, MD: National Institutes of Health (2018). Available online at: https://imagej.nih.gov/ij/ (accessed April 21, 2020).

37. Denney W, Duvvuri S, Buckeridge C. Simple, automatic noncompartmental analysis: the PKNCA R package. J Pharmacokinet Pharmacodyn. (2015) 42:11107. doi: 10.1007/s10928-015-9432-2

38. Murphy P, Wang J, Bhagwat S, Munger J, Janssen W, Wright T, et al. CD73 regulates anti-inflammatory signaling between apoptotic cells and endotoxin-conditioned tissue macrophages. Cell Death Diff. (2017) 24:55970. doi: 10.1038/cdd.2016.159

39. Dowling P. Antimicrobial therapy. In: Eds: Bertone, AL, Hospool, LJI, editors. Equine Clinical Pharmacology. Philadelphia: W.B Saunders Co. (2004). p. 1348 .

40. Adamson, P, Wilson W, Hirsh D, Baggot J, Martin L. Susceptibility of equine bacterial isolates to antimicrobial agents. Am J Vet Res. (1985) 46:447-50.

41. Soehnlein O, Lindbom L. Phagocyte partnership during the onset and resolution of inflammation. Nat Rev Immunol. (2010) 10:427-39. doi: 10.1038/nri2779

42. Rinnovati R, Bonelli F, Tognetti R, Claudio G, Rinaldo F, Veronica M, et al. Effect of repeated arthrocentesis on cytology of synovial fluid. J Equine Vet Sci. (2017) 57:112-5. doi: 10.1016/j.jevs.2017.07.008

43. Nocera I, Vitale V, Poli A, Bonelli F, Sgorbini M. Comparison between synovial fluid cytology and joint capsule histopathology in horses with chronic osteochondritis dissecans. Large Anim Rev. (2019) 25:231-4.

44. Walton RM, Cowell RL, Valenciano AC. Equine Hematology, Cytology, and Clinical Chemistry. Hoboken, NJ: Wiley-Blackwell (2020).

45. Anderson JR, Phelan MM, Clegg PD, Peffers MJ, RubioMartinez LM. Synovial fluid metabolites differentiate between septic and non-septic joint pathologies. J Proteome Res. (2018) 17:2735-43. doi: 10.1021/acs.jproteome.8b00190

46. Frisbie D, Cross M, McIlwraith C. A comparative study of articular cartilage thickness in the stifle of animal species used in human pre-clinical studies compared to articular cartilage thickness in the human knee. Vet Comp Orthop Traumatol. (2006) 19:142-6. doi: 10.1055/s-0038-1632990
47. McIlwraith C, Fortier L, Frisbie D, Nixon A. Equine models of articular cartilage repair. Cartilage. (2011) 2:317-26. doi: 10.1177/1947603511406531

48. McIlwraith C, Frisbie D, Kawcak C. The horse as a model of naturally occurring osteoarthritis. Bone Joint Res. (2012) 1:297-309. doi: 10.1302/2046-3758.111.2000132

49. Chu C, Szczodry M, Bruno S. Animal models for cartilage regeneration and repair. Tissue Eng Part B Rev. (2010) 16:10515. doi: 10.1089/ten.teb.2009.0452

50. Reesink H, Watts A, Mohammed HO, Jay GD, Nixon AJ. Lubricin/proteoglycan 4 increases in both experimental and naturally occurring equine osteoarthritis. Osteo Cart. (2017) 25:128-37. doi: 10.1016/j.joca.2016.07.021

51. Frisbie D, Kawcak C. Effects of triamcinolone acetonide on an in vivo equine osteochondral fragment exercise model. Equine Vet J. (1997) 29:34959. doi: 10.1111/j.2042-3306.1997.tb03138.x

52. Gilbertie J, Schnabel L, Hickok NJ, Jacob ME, Conlon BP, Shapiro IM, et al. Equine or porcine synovial fluid as a novel ex vivo model for the study of bacterial free-floating biofilms that form in human joint infections. PLOS ONE. (2019) 14:e0221012. doi: 10.1371/journal.pone.0221012

53. Coakley G, Matthew C, Field M, Jones A, Kingsley G, Walker D, et al. BSR \& BHPR, BOA, RCGP and BSAC guidelines for management of the hot swollen joint in adults. Rheumatology. (2006) 45:103941. doi: 10.1093/rheumatology/kel163a

54. Wright I, Smith M, Humphrey D, Eaton-Evans TCJ, Hillyer MH. Endoscopic surgery in the treatment of contaminated or infected synovial structures. Equine Vet J. (2003) 35:613-9. doi: 10.2746/042516403775467225

Conflict of Interest: The authors declare that the research was conducted in the absence of any commercial or financial relationships that could be construed as a potential conflict of interest.

Copyright (C) 2021 Pezzanite, Chow, Hendrickson, Gustafson, Russell Moore, Stoneback, Griffenhagen, Piquini, Phillips, Lunghofer, Dow and Goodrich. This is an open-access article distributed under the terms of the Creative Commons Attribution License (CC BY). The use, distribution or reproduction in other forums is permitted, provided the original author(s) and the copyright owner(s) are credited and that the original publication in this journal is cited, in accordance with accepted academic practice. No use, distribution or reproduction is permitted which does not comply with these terms. 